\title{
CLASS DEPENDENT COMPRESSIVE-PROJECTION PRINCIPAL COMPONENT ANALYSIS FOR HYPERSPECTRAL IMAGE RECONSTRUCTION
}

\author{
Wei Li, Saurabh Prasad, James E. Fowler, Lori M. Bruce \\ Department of Electrical and Computer Engineering, Geosystems Research Institute, \\ Mississippi State University, USA
}

\begin{abstract}
Random projections have been demonstrated to be an efficient dimensionality reduction technique for Hyperspectral Imagery (HSI). Compressive-Projection Principal Component Analysis (CPPCA) is an efficient receiver-side reconstruction technique that recovers HSI data from encore-side random projections. In this paper, after receiving random projections from the encoder, we utilize a relatively small amount of training (ground-truth) data to partition the image into several subsets (such that each subset represents a unique class/object) in the projected domain, and then employ the CPPCA reconstruction algorithm independently to every group. It is expected that such a class-dependent reconstruction of HSI data will be more reliable, since it is based on statistics that are representative of the dominant mixtures in the scene. Experimental results with HSI datasets reveal that the proposed method is superior in performance compared to traditional CPPCA.
\end{abstract}

Index Terms - dimensionality reduction, random projection, hyperspectral imagery

\section{INTRODUCTION}

Hyperspectral imagery (HSI) provides a dense recording of reflectance values over a wide region of the spectrum. This is often a double edge sword - the availability of this rich spectral information is expected to improve the performance of image analysis techniques; however, this comes at the cost of computational complexity, over-dimensionality and statistical ill-conditioning in the limited training data conditions. Further, high dimensional HSI data also increases costs associated with additional bandwidth required for transmission of data from the remote sensing modality to the on-ground station, data storage costs etc. Compression of HSI data is hence a critical need, and is an active research area in the remote sensing community. Conventional image compression techniques have already been successfully applied to HSI data [1].

Compressed sensing (CS) is a recently developed mathematical paradigm [2] that under certain conditions, exactly recovers signals sampled at sub-Nyquist rates via a linear projection onto a random basis. CS shifts the computational burden of data from the encoder associated random projections to the decoder side. Alternatively, Fowler [3] proposed a novel image reconstruction strategy Compressive-Projection Principal Component Analysis (CPPCA), which is driven by projections at the sensor onto randomly-chosen lower-dimensional vectors. The CPPCA decoder, given only these random projections, recovers not only the coefficients associated with the PCA transform, but also an approximation to the PCA transform basis itself.

Recently, researchers have begun to explore the efficacy of pattern classification methods in random projected subspaces. In [4], the author studies the efficacy of learning Gaussian mixtures when high-dimensional data is randomly projected into low-dimensional subspaces. In [5] the authors demonstrate the applicability of support vector machine (SVM) classifiers in random projected subspaces. In [6], the authors exploit the Johnson-Lindenstrauss (JL) lemma to demonstrate that if a low-dimensional subspace is picked randomly, the cluster separation is approximately retained in the projected subspace.

In this paper, we propose a class-dependent CPPCA algorithm. We use a small quantity of a-priori labeled data, to train a classifier based on linear discriminant analysis (LDA) [7] and Gaussian maximum-likelihood parameter estimation (MLE) [8]. This classification setup is used to build a ground-cover map of the HSI image. After the grouping procedure, the CPPCA algorithm is employed on each class independently. The motivation behind this work is that a class-dependent CPPCA will recover the principle eigenvectors most suited for each class separately, and in doing so, the overall decoder-side signal reconstruction from encoder-side random projections will be more accurate.

This paper is organized as follows. In section 2, we describe the supervised classification strategy employed in this paper. In section 3, we provide a description of the CPPCA algorithm and the proposed class-dependent CPPCA procedure. In section 4, using HSI datasets, we compare the performance of proposed method with conventional CPPCA. We conclude the paper with a summary and future work in section 5 . 


\section{SUPERVISED CLASSIFICATION}

The supervised classification strategy employed in this paper consists of LDA based dimensionality reduction followed by Gaussian quadratic maximum-likelihood classification. LDA seeks to find a linear transformation $\varphi$ which projects the data from a high dimensional space onto a lower dimensional subspace, by minimizing the within-class scatter and maximizing the between-class scatter (and hence maximizing class separation in the projected subspace). The LDA solution is obtained by maximizing Fisher's ratio [7],

$$
J(\varphi)=\frac{\varphi^{T} S_{b} \varphi}{\varphi^{T} S_{w} \varphi},
$$

where $S_{b}$ is the between-class scatter matrix and $S_{w}$ is the within-class scatter matrix. The maximizing solution is obtained by solving the following generalized eigenvalue problem

$$
S_{b} \varphi=\Lambda S_{w} \varphi,
$$

where $\Lambda$ is the diagonal eigenvalue matrix.

The dimensionality of the projected subspace after an LDA transformation is upper bounded to $c-1$ by design ( $c$ is the number of classes in the classification task). A detailed discussion of LDA can be found in [7]. Following LDA based dimensionality reduction, a Gaussian quadratic maximum-likelihood classifier is employed to generate a ground-cover map of the HSI data.

\section{CPPCA RECONSTRUCTION}

In the algorithm proposed in this paper, we focus on CPPCA instead of CS because in previous work [3], CPPCA has been found to outperform CS for decoder-side reconstruction. CPPCA is driven by projections at the sensor onto randomly-chosen lower dimensional sets of vectors. The use of random projections at the encoder-side eliminates the need for computationally complex dimensionality reduction. The decoder, given only these random projections, recovers not only the coefficients associated with the PCA transform, but also an approximation to the PCA transform basis itself. Consider $M$ zero-mean vectors $X=\left[x_{1}, x_{2}, \cdots, x_{M}\right]$, where $X \in \mathfrak{R}^{N}$. The covariance matrix of $X$ is $\Sigma_{X}=X X^{T} / M$, and PCA seeks to find a linear transformation $W$ of eigenvectors emanating from the eigen-decomposition of $\Sigma_{X}$; i.e.,

$$
\Sigma_{X}=W \Lambda W^{T}
$$

where $W$ contains the $d$ unit eigenvectors of $\Sigma_{X}$ columnwise. The PCA transform of $X$ is then $\breve{X}=W^{T} X$.

CPPCA reconstruction uses the first $L$ Ritz vectors [9], which are calculated from $\tilde{\Sigma}_{\tilde{Y}}=P^{T} \Sigma_{X} P$ ( $P$ is the projection matrix with size $N \times K, K \ll N$ ), to obtain an approximation of the first $L$ principal eigenvectors in $W$. These approximation eigenvectors are assembled into a $N \times L$ matrix $\Psi$. The reconstruction of $X$ is then produced in a pseudoinverse-based recovery of the PCA coefficients,

$$
\hat{X}=\Psi\left(P^{T} \Psi\right)^{\dagger} \tilde{Y} .
$$

The reader is referred to [3] for greater detail.

In the proposed approach, the CPPCA decoding algorithm is employed independently for every class based on the ground-cover classification obtained using encoder-side projections of the HSI data. In every group (class), the direction of the dominant eigenvector is the direction of maximum variance of samples (pixels) in that class. It is expected that the proposed class-dependent CPPCA will be more accurate at the decoding task than conventional CPPCA. This can be attributed to multiple modes in the data-distribution arising from the presence of multiple classes/objects in the image. Hence, this class-dependent CPPCA will employ statistics pertinent to each class as opposed to the average statistics over all classes. The resulting decoder hence exploits "local" individual geometrical distributions as provided by the covariance matrix of each class as opposed to a single aggregated distribution employed in conventional CPPCA.

Hence, at the decoder, we first employ a supervised classification method to assign a class label to all pixels in the image using data in the random projected subspace. This is followed by a CPPCA based reconstruction from random projections for each class separately. The direction of the dominant eigenvectors (i.e. those with the largest associated eigenvalues) of a class is the direction of maximum variance of samples in that class. The motivation for this work is that CPPCA will recover the principal eigenvectors that are representative of the statistics of each class. We hence expect the reconstruction procedure to be more precise when CPPCA is applied separately for each class instead of when it is applied on the entire dataset.

\section{EXPERIMENTAL RESULTS}

We demonstrate and quantify the efficacy of the proposed approach using two HSI datasets - representing a typical urban scene and a vegetation characterization and precision agriculture problem respectively. The first experimental hyperspectral imagery employed in this work covers the Washington DC Mall area [10]. This aerial image represents an urban scenario with $1280 \times 307$ pixels and 191 spectral bands in the 0.4 to $2.4 \mu \mathrm{m}$ region of the visible and infrared spectra acquired by the HYDICE sensor. In this experiment, training data from five dominant ground-cover classes are extracted (Roofs, Streets and Paths, Grass, Trees, and Water).

The second dataset we used is an aerial hyperspectral image acquired with the Pro-SpecTIR-VNIR ${ }^{\mathrm{TM}}$ sensor. This 
dataset was collected over an experimental corn field at Mississippi State University in Brooksville, MS on June 6, 2008. The image dimensions are $283 \times 213$ pixels with 128 spectral bands in the 0.4 to $0.994 \mu \mathrm{m}$ region. This dataset represents corn crop under varying degrees of chemical induced stress - each degree of stress in the crop is a unique class in this dataset (for a total of three classes).

Figs. 1-2 illustrate the classification performance (overall classification accuracy) in the randomly projected domain for both datasets. Input features to the classifiers comprise of the HSI imagery after being projected onto a lower dimensional random subspace (to simulate encoder-side random projections in a traditional deployment of CPPCA). For the first dataset, approximately 2,000 training pixels (approximately 400 samples per class) and 6,000 testing pixels (approximately 1,200 samples per class) are employed. For the second dataset, approximately 1,300 training pixels (approximately 400 samples per class) and 1,300 testing pixels (approximately 400 samples per class) are employed. It is clear from these results that performing reliable ground-cover classification in the randomly projected subspace is indeed possible. In fact, we found that the classification accuracy in the random subspace domain (with a 50\% reduction in dimensionality) was slightly higher than that of the same classifier in the raw spectral domain with no projections. This is expected to be an artifact of the over-dimensionality in the original HSI space coupled with limited training data.

Figs. 3-4 illustrate the corresponding classification maps obtained by using this limited training data using encoderside random projections. Each color represents a unique class in this ground-cover map. In the proposed method, the class-dependent CPPCA decoder performs the decoding/reconstruction for each class independently.

Performance of the proposed decoder is quantified by reporting the vector-based signal-to-noise ratio (SNR) measured in dB. From Figs. 5-6, we see that the proposed method yields an average SNR substantially higher than that of conventional CPPCA over a broad range of practical subrates (defined as $K / N$ ). For the DC Mall dataset, the algorithm achieves a 2-12 dB improvement in SNR. For the corn field dataset, the proposed method provides a 1-2 $\mathrm{dB}$ average gain. The relatively higher gain with the first dataset can be attributed to the fact that the various "classes" in the scene were indeed very distinct, as opposed to the second dataset where classes were representing the same species of a crop under different degrees of stress.

\section{CONCLUSION}

In this paper, we proposed a hyperspectral image reconstruction approach which combines supervised classification with the recently proposed CPPCA reconstruction algorithm. We employed a simple classifier
(LDA+MLE) to partition the HSI data using the encoderside projections into distinct classes. CPPCA is then applied to each class individually. The proposed method consistently outperformed conventional CPPCA and is hence highly suitable for HSI reconstruction from random projections. Further, in the absence of ground-truth (labeled) data for training this system, an unsupervised clustering method can also be employed in this framework where the CPPCA decoding is performed on each cluster independently.

\section{REFERENCES}

[1] Q. Du and J. E. Fowler, "Hyperspectral image compression using JPEG2000 and principal component analysis", IEEE Geoscience and Remote Sensing letters, vol. 4, no. 2, pp. 201-205, April 2007.

[2] E. Candes, J. Romberg, and T. Tao, "Robust uncertainty principles: Exact signal reconstruction from highly incomplete frequency information", IEEE Transactions on Information Theory, vol. 52, no. 2, pp. 489-509, February 2006.

[3] J. E. Fowler, "Compressive-projection principal component analysis," IEEE Transaction on Image Processing, vol. 18, no. 10, pp. 2230-2242, October 2009.

[4] S. Dasgupta, "Experiments with random projection," in Proceedings of the 16th Conference on Uncertainty in Artificial Intelligence, pp. 143-151, San Francisco, CA, 2000.

[5] R. Calderbank, S. Jafarpour, and R. Schapire, "Compressed learning: Universal sparse dimensionality reduction and learning in the measurement domain," Manuscript, 2009.

[6] R. Baraniuk and M. Wakin, "Random projections of smooth manifolds," Foundations of Computational Math., 2007.

[7] Duda, R. O., Hart, P. E. and Stocrk, D. G., Pattern Classification. John-Wiley and Sons, New York, NY, 2001.

[8] S. D. Zenzo, S. D. Degloria, R. Bernstein and H. C. Kolsky, "Gaussian maximum likelihood and contextual classification algorithm for multicrop classification," IEEE Transactions on Geoscience and Remote Sensing, vol. 25, pp. 805-814, November 1987.

[9] B. N. Parlett, The Symmetric Eigenvalue Problem. Philadelphia, PA: SIAM, 1998.

[10] D. Landgrebe, "Hyperspectral image data analysis as a high dimensional signal processing problem," IEEE Signal Processing Magazine, vol. 19, no. 1, pp. 17-28, January 2002.

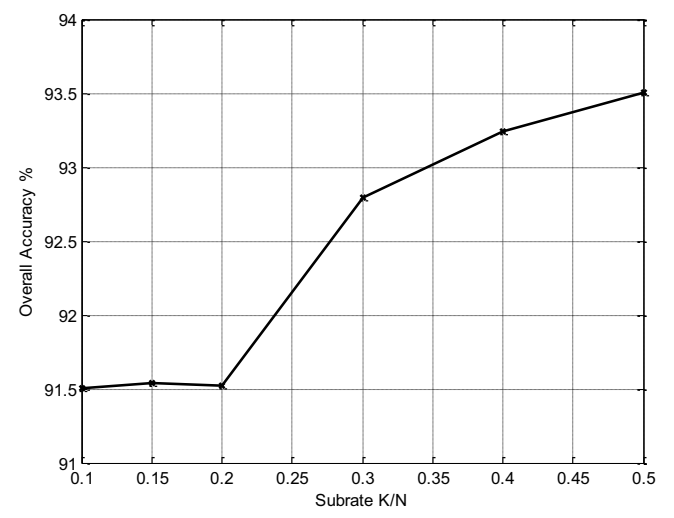

Fig. 1. Overall classification accuracy versus subrate in the random subspace domain for the DC Mall dataset. 


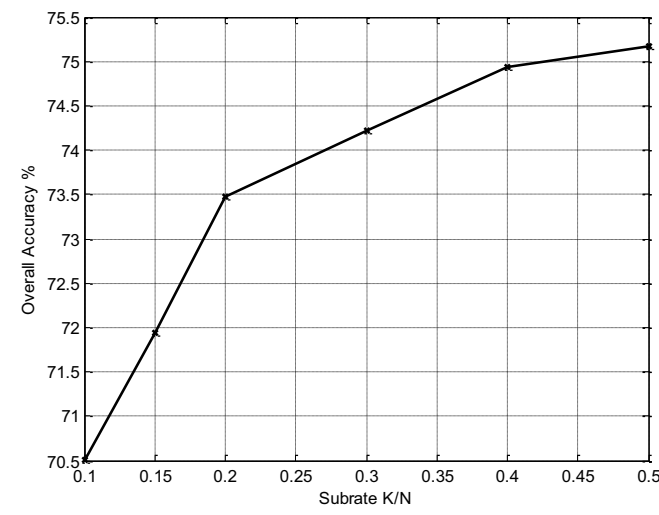

Fig. 2. Overall classification accuracy versus subrate in the random subspace domain for the corn field dataset.
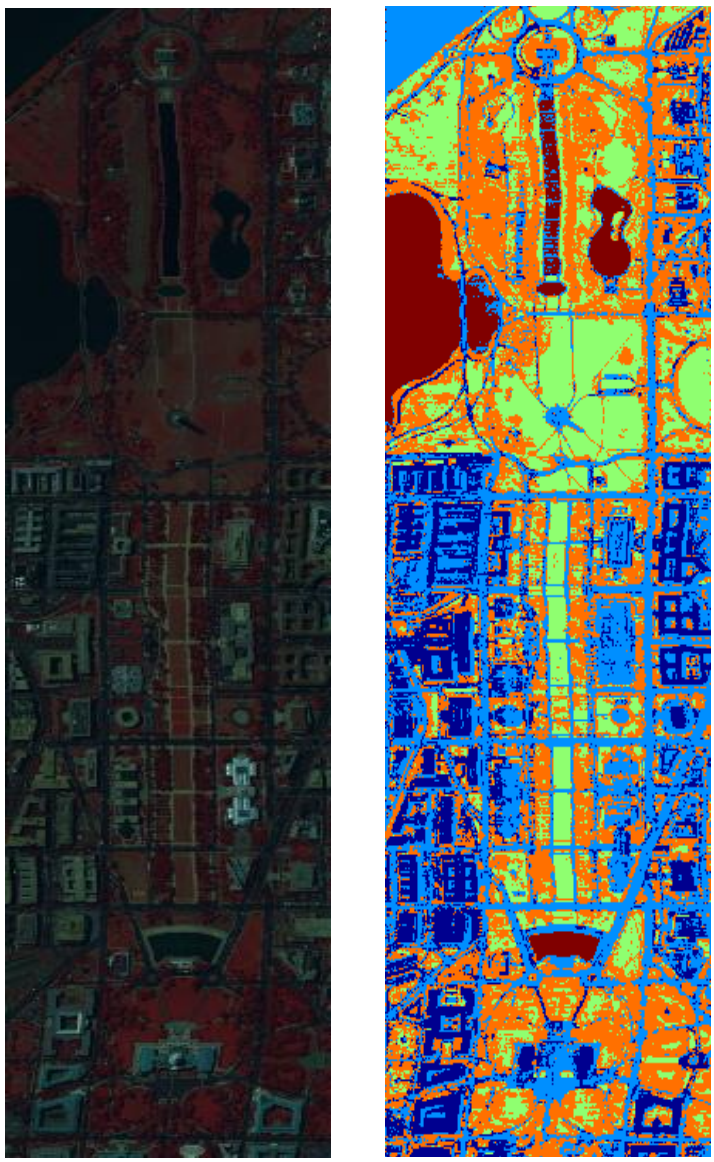

Fig. 3. (left) pseudo-color image of the DC Mall dataset; (right) classification map using an LDA-MLE classifier.
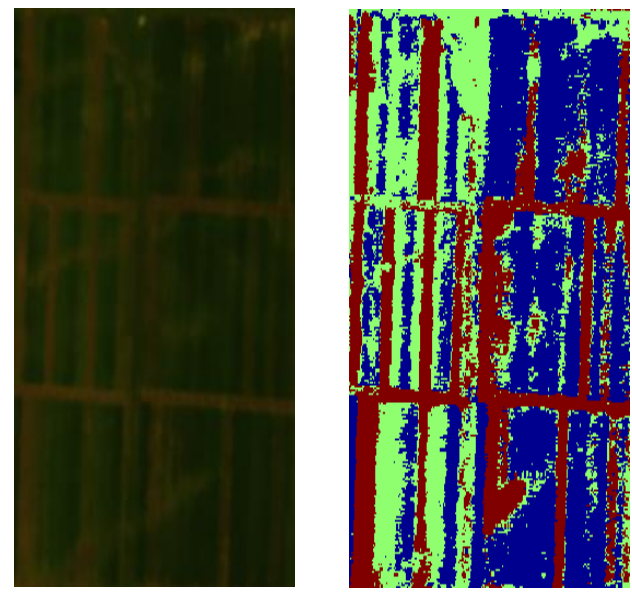

Fig. 4. (left) original RGB-color image of the corn field dataset; (right) classification map using an LDA-MLE classifier.

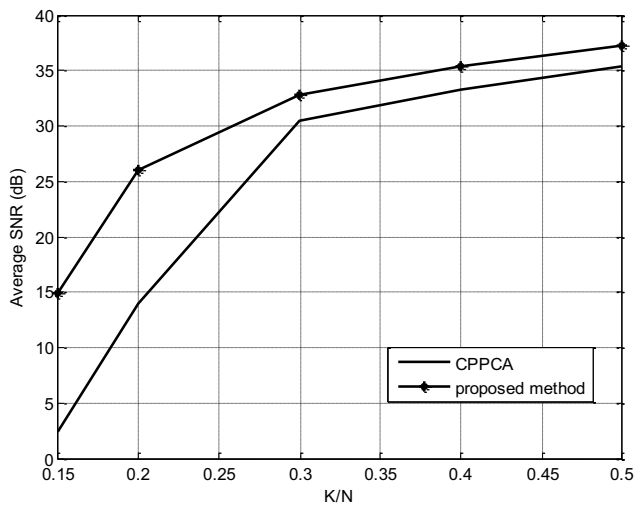

Fig. 5. Average SNR versus subrate for the DC Mall dataset.

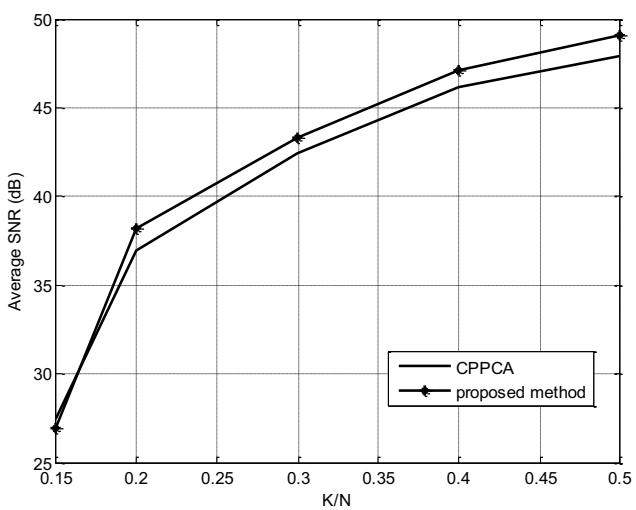

Fig. 6. Average SNR versus subrate for the corn field dataset. 